\title{
An Underwater Sensor Allocation Scheme for Noncircular Sensing Coverage Regions
}

\author{
Erik F. Golen, ${ }^{1}$ Carl V. Lutzer, ${ }^{2}$ David S. Ross, ${ }^{2}$ and Nirmala Shenoy ${ }^{1}$ \\ ${ }^{1}$ Laboratory for Wireless Networking and Security, Rochester Institute of Technology, Rochester, NY 14623, USA \\ ${ }^{2}$ School of Mathematical Sciences, Rochester Institute of Technology, Rochester, NY 14623, USA \\ Correspondence should be addressed to Erik F. Golen; efg5752@rit.edu
}

Received 4 December 2012; Accepted 26 December 2012

Academic Editors: T.-S. Chen and B. Tavli

Copyright (C) 2013 Erik F. Golen et al. This is an open access article distributed under the Creative Commons Attribution License, which permits unrestricted use, distribution, and reproduction in any medium, provided the original work is properly cited.

\begin{abstract}
Intelligently allocating underwater sensors to a large area of interest whose acoustic characteristics vary throughout is a challenge, especially for an area clearance scenario. In these scenarios, there is no apparent target for an adversary to gravitate towards, such as a ship or a port. Thus, it is difficult to determine how the field designer should allocate sensors so that their deployment locations can be planned efficiently. The previously proposed Game Theory Field Design (GTFD) model can achieve an intelligent sensor allocation, using a game theoretic approach, for sensors with circular coverage regions. In practice, however, the sensing coverage of an underwater sensor will likely be noncircular due to the azimuthally dependent bathymetric phenomena and other underwater irregularities. As a result, an extension of the model is proposed for allocating sensors for the irregularly shaped sensing coverage regions. This work provides two validations of the extended GTFD model. The first is an analytical comparison with sensing coverage regions whose shape is well understood, and the second uses simulation to validate the model for the irregularly shaped regions.
\end{abstract}

\section{Introduction}

When designing an underwater sensor field for an area clearance scenario, a field designer's responsibility is to create a sensor field whose purpose may range from preventing the deployment of mines to averting illegal traversal and surveillance of a restricted water space. Sensor allocation to an area of interest (AOI) in an area clearance scenario is complex, as there are no obvious targets that an adversary would gravitate towards, such as a port [1]. Thus, the field designer is left to blindly guess as to how to allocate a fixed number of available sensors, unless an analysis of the acoustic characteristics of the $\mathrm{AOI}$ is done.

The underwater environment introduces challenges that are not experienced terrestrially, such as signal transmission loss due to geometric spreading and absorption by the ocean [2-4]. Additionally, multipath, as well as man-made and ambient noise, can cause significant interference [24]. As a result, the sensing range of a sensor is limited, making underwater vehicle detection quite difficult [2-4]. Additionally, sound speed, and as a result, transmission loss, are dependent upon water temperature, depth, salinity, and time of year. Furthermore, transmission loss, and in turn, sensing range, is range dependent, meaning that it varies by a sensor's physical location $[4,5]$.

Since the underwater environment exhibits range dependence, it is possible to subdivide an AOI into regions of relatively uniform acoustic characteristics, or sectors [5]. Once these sectors have been determined, sensor allocation can be performed using the Game Theory Field Design (GTFD) model. This model, proposed in [5], uses a game theoretic approach to determine probabilistically how often an intelligent adversary will visit each sector. The allocation is based upon an assumed speed and length of mission, along with the geographic size of each sector and its corresponding acoustics. However, only circular sensing coverage regions are considered in this model.

Due to the azimuthally dependent bathymetric phenomena, such as slopes and shelves, and other irregularities often experienced underwater, it is highly likely that a sensor's coverage will not be circular [4]. Thus, an extension of the GTFD model is required to compute the probabilistic 
visitation of an adversary to sectors within an AOI for irregularly shaped sensing coverage regions. This extension, along with an analytical and simulation-based validation of the approach, is the principal contribution of this work.

The remainder of this paper is organized as follows. First, a discussion of search theory used in the GTFD model is given in Section 2, followed in Section 3 by the model extension used to consider irregularly shaped sensing coverage regions. An analytical validation of the approach is provided in Section 4, and a simulation-based validation is shown in Section 5. Conclusions are drawn in Section 6.

\section{Search Theory}

A main component of the GTFD model is a minimax matrix game that is solved in order to determine an adversary's probability of visitation to sectors of relatively uniform acoustic characteristics. In order to quantify how the matrix game is populated, a discussion of search theory is required.

In [5], based upon acoustic characteristics in the AOI, a circular sensing coverage region for a single sensor with sensing radius $r$ is derived for each sector. The probability of detection $\mathrm{PD}(t)$ for a single sensor within a sector of geographic area $A$ against an adversary traveling at an average speed $v_{\text {eff }}$ for some time $t$ can be calculated using the search equation derived by Koopman in [6]

$$
\mathrm{PD}(t)=1-e^{-W L / A} \text {. }
$$

The standard definition of (1) dictates that a searcher with a sweep width $W$ traverses a region of area $A$ for some distance $L$, where $L$ is the product of the sensor's average speed and the amount of time spent searching, $v_{\text {eff }}$ and $t$, respectively [7]. The sweep width can be thought of as a broom sweeping the region, where any adversary that comes into contact with the broom is assumed to be "swept up," or detected, with a probability of 1 . Conversely, adversaries not swept up are detected with a probability of 0 [7]. In this work, sensors are stationary, while the adversary is mobile, thus the average speed of the sensor is called $v_{\text {eff }}$ since its speed is relative to the adversary.

For the circular sensing coverage case, rotating an infinitesimally thin "broom" that has a length of $2 r$ forms a circular sensing coverage region of sweep width $2 r$ [7]. However, for noncircular sensing coverage regions, the sweep width of that coverage region must be calculated using the method shown in Section 3. The exponential in (1) is a ratio of the search effort by the searcher to the area of the region $A$ and is the probability of missing the adversary [7]. As a result, (1) represents the probability of detection of the adversary by a single sensor.

Knowing the probability of detection for a single sensor allows for an extended use of Koopman's search equation for multiple sensors, which is the cumulative probability of the detection $\mathrm{CPD}(t)$ for some number of sensors, NS, of located within a sector [6]

$$
\mathrm{CPD}(t)=1-(1-\mathrm{PD}(t))^{\mathrm{NS}}
$$

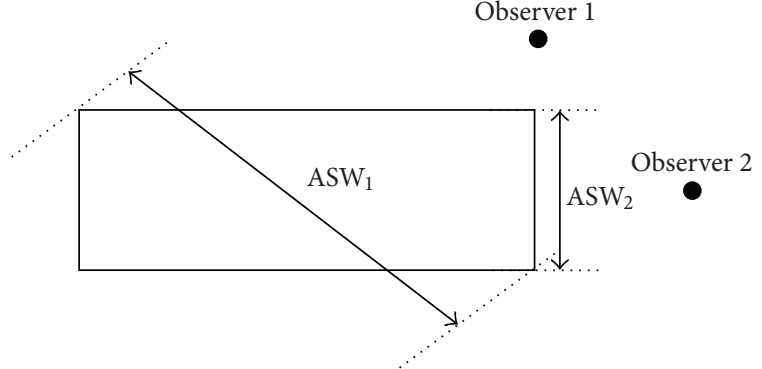

FIGURE 1: Apparent sweep width perceived by observer $k$ is $\mathrm{ASW}_{k}$.

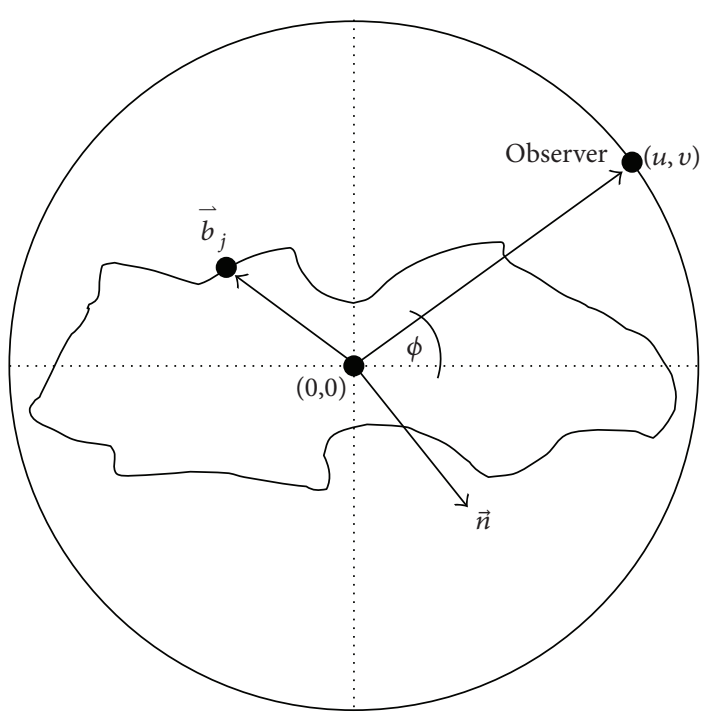

FIGURE 2: Apparent sweep width of a general-shaped region.

Equation (2) implies that the sensing coverage region of each sensor is not only independent, but also nonoverlapping, allowing the "miss" probability of a single sensor to be raised to the number of sensors in the sector. This computation of $\mathrm{CPD}(t)$ for each sector becomes the basis for the matrix game in the GTFD model [5].

\section{Sweep Width Calculation}

To an observer, such as a submarine, a circular sensing coverage region appears to have a sweep width of $2 r$, where $r$ is the sensing radius of the coverage region, regardless of the vantage point of the observer.

However, for a noncircular sensing coverage region, the apparent sweep width (ASW) of the region differs depending upon the location of the observer with respect to the region. Figure 1 shows that observers at different locations perceive a significantly different ASW of the same rectangular region. This is captured in the aggregate by calculating the mean ASW (also called the sweep width). This calculation is described below.

Figure 2 shows a general sensing coverage region, centered at the origin, and a single observer at $(u, v)$ that is separated from the horizontal axis by an angle of $\phi$ radians. 
TABLE 1: Comparison of sweep width for circular region.

\begin{tabular}{lccccc}
\hline$r(\mathrm{kyd})$ & $W_{\text {circ }}(\mathrm{kyd})$ & $W_{R} 0.1 \mathrm{kyd}^{2}$ & Error $(\%)$ & $W_{R} 1 \mathrm{kyd}^{2}$ & 13.02 \\
\hline 6.9 & 13.8 & 13.92 & 0.87 & 16.89 & Error $\left.^{2} \%\right)$ \\
8.9 & 17.8 & 17.85 & 0.28 & 22.61 & 5.65 \\
11.5 & 23 & 22.99 & 0.04 & 29.84 & 11 \\
15 & 30 & 30.11 & 0.37 & 39.19 & 0.53 \\
19.7 & 39.4 & 39.57 & 0.43 & 46.75 & 0.53 \\
23.5 & 47 & 47.17 & 0.36 & 0.53 \\
\hline
\end{tabular}

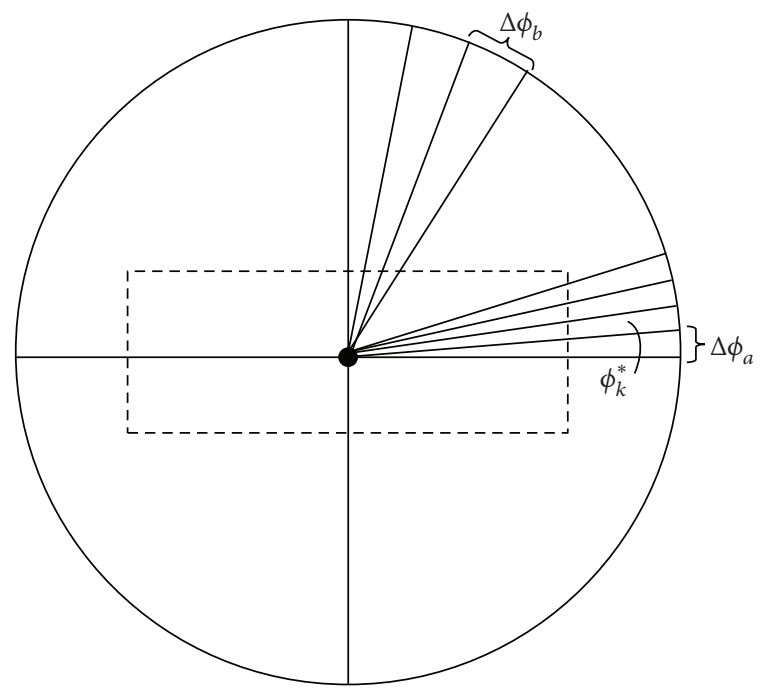

FIGURE 3: Discrete calculation of sweep width.

We define $\mathbf{n}$ to be a unit vector orthogonal to $(u, v)^{T}$, and for each boundary point, the scalar projection $r=\mathbf{n} \bullet \mathbf{b}$ is calculated, where $\mathbf{b}$ is the boundary point's position vector. After calculating the scalar projection for each boundary point, we define the ASW in (3) as

$$
\operatorname{ASW}(\phi)=\max _{j}\left(r_{j}\right)-\min _{j}\left(r_{j}\right) .
$$
ASW

The sweep width $W$ of the region in (4) is the average

$$
W=\frac{1}{2 \pi} \int_{0}^{2 \pi} \operatorname{ASW}(\phi) d \phi,
$$

which is approximated with a Riemann sum

$$
W_{R} \approx \frac{1}{2 \pi} \sum_{k=1}^{n} A S W\left(\phi_{k}^{*}\right) \Delta \phi_{k} .
$$

The sampling angles, $\phi_{k}^{*}$, and the associated step sizes $\Delta \phi_{k}$ in (5) are determined as follows.

We begin by dividing the detection region into pixels of a given size. Each point on the boundary of the detection region defines a direction from which an observer can approach the detector, $\phi_{k}^{*}$, and the discretization of the boundary dictates the values of $\Delta \phi_{k}$. An example is shown in Figure 3, wherein the detector is at the origin, and the detection region is rectangular. Note that boundary points on the right and left sides of the detection region are farther from the detector than those along the top of the shape. Consequently, the angular step size between them is smaller $\left(\Delta \phi_{a}<\Delta \phi_{b}\right)$.

As indicated above, each boundary point is associated with a direction of approach. Specifically, when boundary point $k$ has Cartesian coordinates $x_{k}$ and $y_{k}$, we define

$$
\phi_{k}^{*}=\tan ^{-1}\left(\frac{y_{k}}{x_{k}}\right) .
$$

The associated step size for a boundary point $k$ is calculated using the difference between the sampling angle of the adjacent boundary point counterclockwise to it, $k+1$. That is,

$$
\Delta \phi_{k}=\left|\phi_{k+1}^{*}-\phi_{k}^{*}\right|
$$

\section{Analytical Validation}

Presented in this section is an analytical validation of the sweep width calculation $W_{R}$ in (5). Comparisons of the calculated sweep width are made with the theoretical sweep widths for two well-understood shapes, a circle and a rectangle, for two pixel sizes, 0.1 and $1 \mathrm{kyd}^{2}$. As mentioned, the sweep width of a circle $W_{\text {circ }}$ is calculated as $2 r$, where $r$ is the radius of the circle.

Table 1 shows the theoretical sweep width of a circular sensing coverage region $W_{\text {circ }}$ and the calculated sweep width $W_{R}$ with sensing radius $r$ between 6.9 and $23.5 \mathrm{kyd}$ for pixel sizes of 0.1 and $1 \mathrm{kyd}^{2}$, along with the associated error for each calculation. As the pixel size increases, the error in the calculation also increases; error decreases with larger sensing radius because more boundary points exist.

Since we expect few, if any, sampling problems in the case of a circle, due to the even intervals of boundary points, the sweep width of a rectangle is also investigated to validate the effectiveness of the weighting factor used. Using (4), it can be shown that the sweep width of a rectangle is

$$
W_{\text {rect }}=\frac{4(a+b)}{\pi},
$$

when its sides have lengths of $2 a$ and $2 b$ (as in Figure 3). In Table 2 , seven different rectangle sizes are considered, where $b$ is fixed at $40 \mathrm{kyd}$ and $a$ is varied from 4 to $40 \mathrm{kyd}$.

As before, the error in sweep width calculations increases with increased pixel size but decreases with increased area of 
TABLE 2: Comparison of sweep width for rectangular region.

\begin{tabular}{|c|c|c|c|c|c|c|}
\hline$a(\mathrm{kyd})$ & Ratio $a / b$ & $W_{\text {rect }}(\mathrm{kyd})$ & $W_{R} 0.1 \mathrm{kyd}^{2}$ & Error (\%) & $W_{R} 1 \mathrm{kyd}^{2}$ & Error (\%) \\
\hline 4 & 0.1 & 56.02 & 55.99 & 0.054 & 55.57 & 0.803 \\
\hline 10 & 0.25 & 63.66 & 63.65 & 0.016 & 63.5 & 0.251 \\
\hline 13.2 & 0.33 & 67.74 & 67.73 & 0.015 & 67.38 & 0.531 \\
\hline 20 & 0.5 & 76.39 & 76.39 & 0 & 76.37 & 0.026 \\
\hline 26.4 & 0.66 & 84.54 & 84.54 & 0 & 84.03 & 0.603 \\
\hline 30 & 0.75 & 89.13 & 89.13 & 0 & 89.13 & 0 \\
\hline 40 & 1 & 101.86 & 101.86 & 0 & 101.85 & 0.010 \\
\hline
\end{tabular}

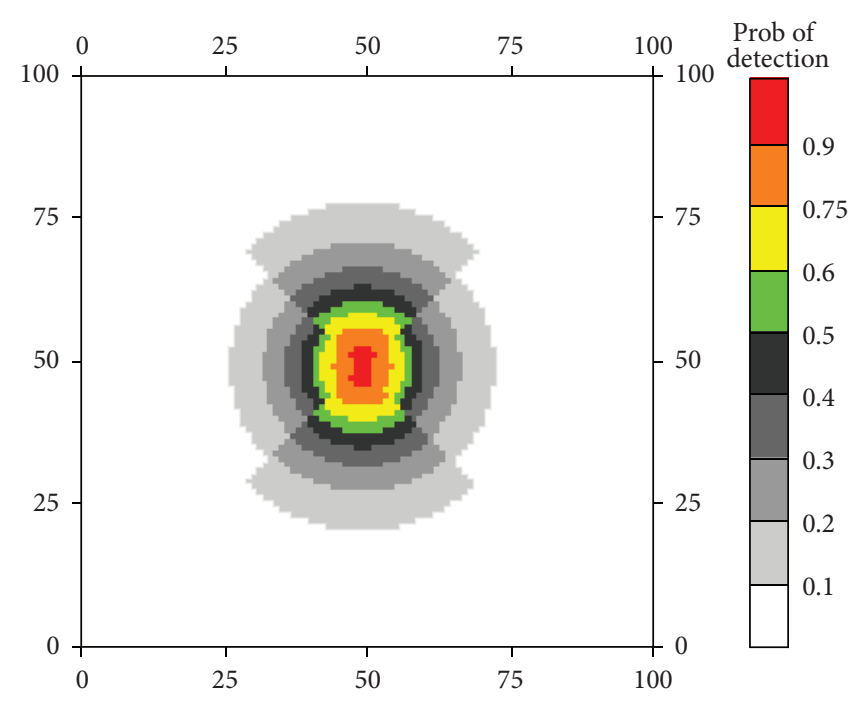

Figure 4: Sensor Region 1.

the rectangle. Note that the error in sweep width calculations for a rectangle is significantly smaller than for the circle case. This happens principally because the number of boundary points available for the rectangles was far greater than that for the circles, resulting in an improved accuracy in the calculations. For example, with a pixel size of $1 \mathrm{kyd}^{2}$, the number of boundary points for the circle calculations ranged from 36 to 132, while there were 176 to 320 boundary points available for the rectangle calculations. More specifically, using a larger number of boundary points tends to result in a smaller $\Delta \phi_{k}$, which attenuates the error in the approximation of $W_{R}$ with the Riemann sum.

\section{Simulation-Based Validation}

Further validation of the approach is provided in this section, where 3 irregularly shaped sensing coverage regions are considered. First, the sweep width of each region was calculated using $0.1 \mathrm{kyd}^{2}$ pixels, and the $\mathrm{PD}(t)$ of a single sensor was determined using (1). Next, the $\operatorname{CPD}(t)$ was calculated for 1 up to 12 sensors, using (2). Finally, these results were compared with simulations done using the Monte Carlo simulator MUSCIAL [8].

Sensing Region 1 , shown in the center of a 100 by $100 \mathrm{kyd}$ AOI in Figure 4, has a range of $11.5 \mathrm{kyd}$ to the north and south, but only $8.9 \mathrm{kyd}$ to the east and west. Using (5), we approximate its sweep width to be $21.25 \mathrm{kyd}$. Based on this calculation and (2), the $\mathrm{CPD}(t)$ (denoted as "Koopman" in the plots below) was determined for groups of sensors whose sensing coverage did not overlap, against adversaries patrolling for 12 hours at 5 knots. Then, MUSICAL was used to simulate this sensing region against 5000 randomly placed adversaries, whose course changed randomly every hour, traveling at 5 knots, for 12 hours. During each simulation, the number of the uniquely detected adversaries was counted to determine the simulated CPD (denoted as "Simulation" in the plots below).

In Figure 5, the CPD values for the "Koopman" curve closely coincide with that of the "Simulation" curve, regardless of the number of sensors in the field. A 95\% confidence interval of the difference between the models was $(-0.167,0.207)$, meaning that there is no statistically significant difference with 95\% confidence between the analytically determined CPD and the simulation, as zero is included in the interval. Additionally, a $P$-value of 0.839 was calculated for the difference of the two means, which far exceeds the value of 0.10 required to show that the two "processes" are not different.

Figure 6 depicts Sensing Region 2, where the sensor has a detection range of $23.5 \mathrm{kyd}$ north and south, but only $6.9 \mathrm{kyd}$ in other directions. This could represent a sensor placed in 


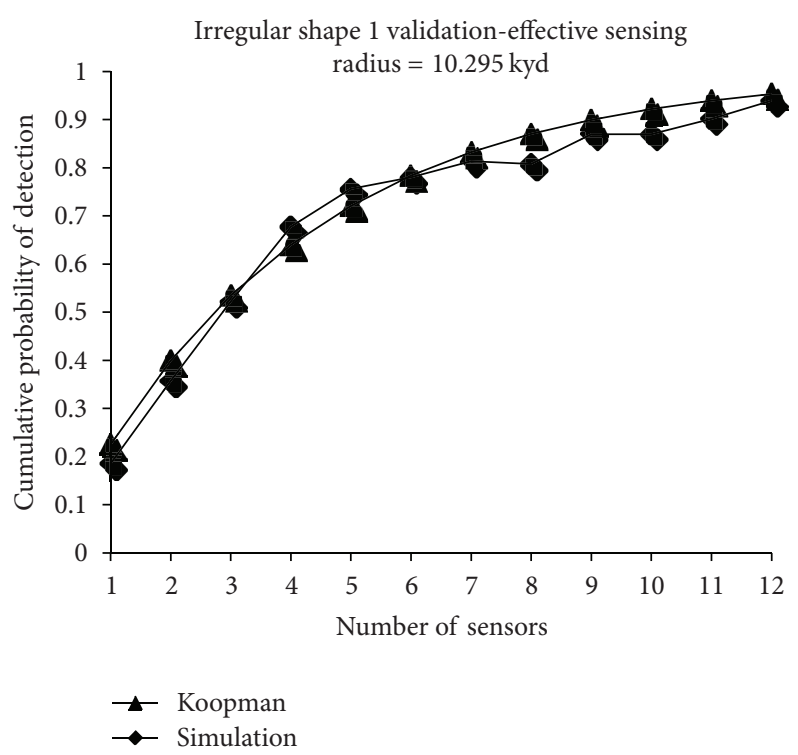

Figure 5: Validation for Sensing Region 1.

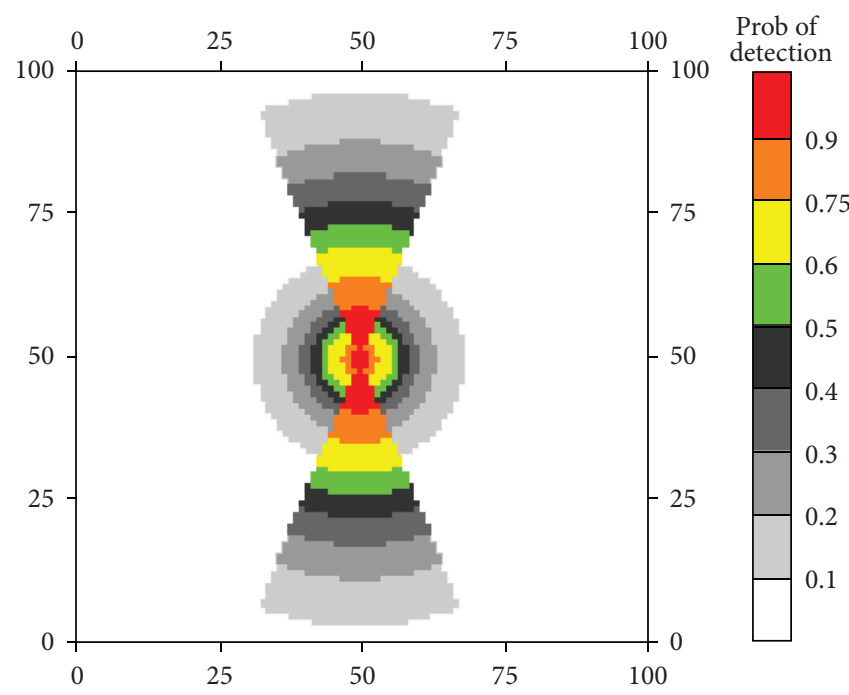

FIgURE 6: Sensing Region 2.

Irregular shape 2 validation-effective sensing radius $=13.254 \mathrm{kyd}$

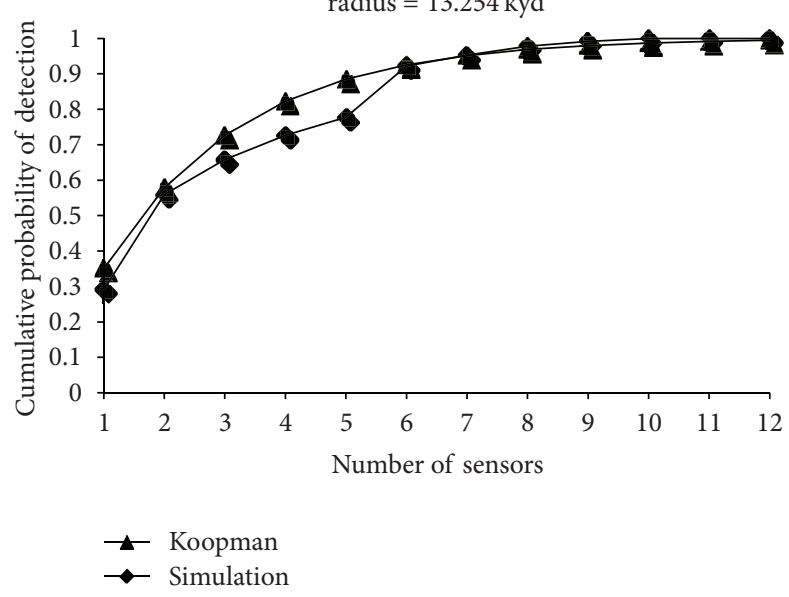

Figure 7: Validation for Sensing Region 2. 


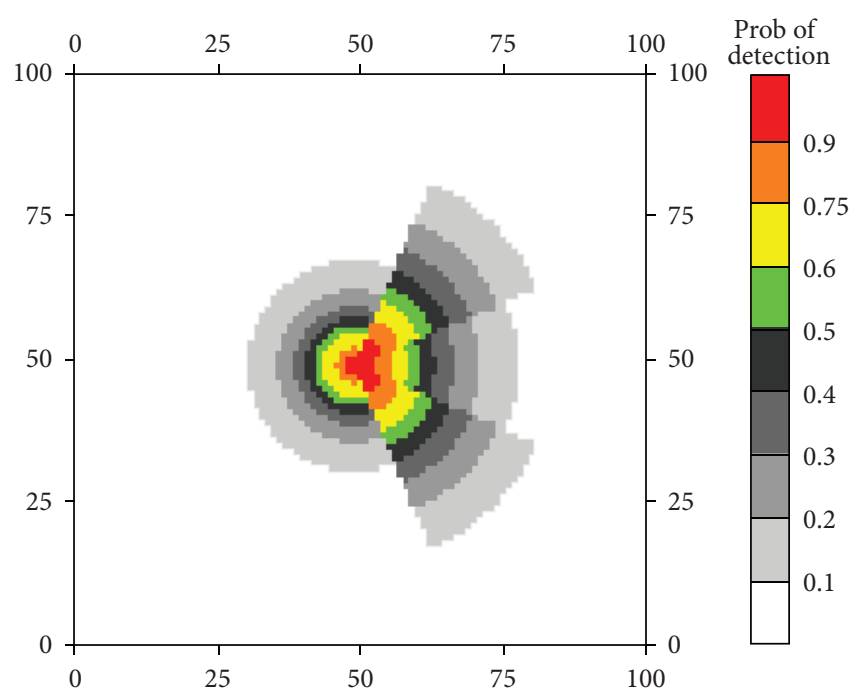

Figure 8: Sensing Region 3.

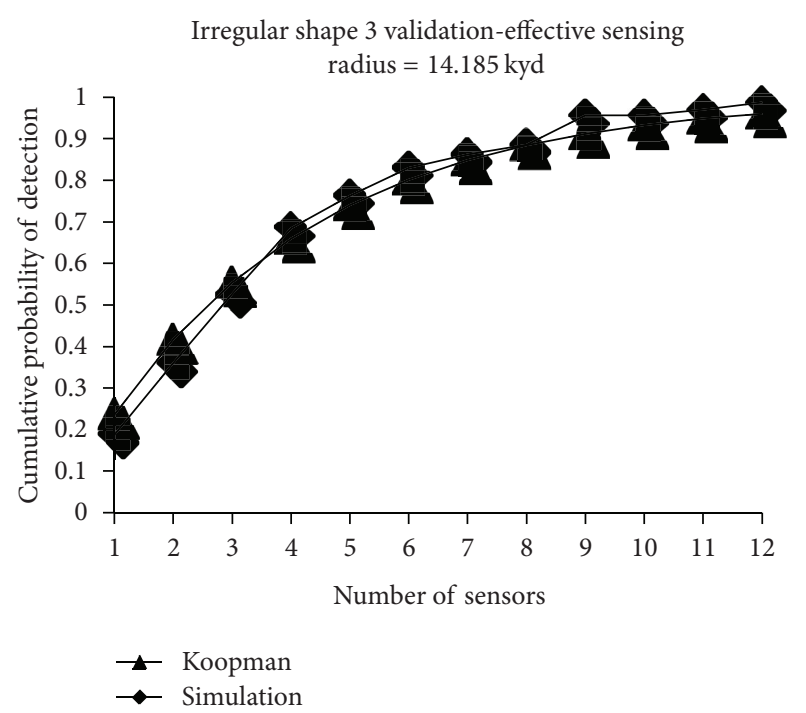

Figure 9: Validation for Sensing Region 3.

a chasm that rises up on the east and west and remains flat to the north and south. The result is an elongated sensing region with a sweep width of $36.01 \mathrm{kyd}$.

For Sensing Region 2, the analytical and simulation results in Figure 7 again closely coincided, with a $P$-value of 0.775 and a $95 \%$ confidence interval of $(-0.146,0.197)$. Even for the first six points on the plot, where there is a detectable difference between the two processes, the $P$-value was 0.682 .

Sensing Region 3, in Figure 8, represents a sensor that can sense out to $15,11.5$, and $15 \mathrm{kyd}$ to the northeast, east, and southeast, respectively, and to $6.9 \mathrm{kyd}$ elsewhere. This suggests that the sensor could be on top of a shelf that drops off to the west.

Lastly, Figure 9 shows that the analytical and simulated results for Sensing Region 3 coincide well. In this case, the $P$-value was 0.953 , and the $95 \%$ confidence interval was $(-0.204,0.191)$.
The results shown in this section prove the validity of the sweep width calculation method presented in Section 3 for irregularly shaped sensing coverage regions, as the $P$ values were all well above 0.1 and the $95 \%$ confidence interval included 0 for all three sensing coverage regions.

\section{Conclusions}

This work has provided a highly accurate method for calculating the sweep width of an irregularly shaped sensing coverage region. The calculated sweep width can then be directly incorporated into the Game Theory Field Design model used to intelligently allocate underwater sensors for area clearance scenarios. Two methods of validation were provided for the aforementioned approach. First, analytically determined sweep widths for both circles and rectangles of varying sizes were compared with the sweep widths calculated using the proposed approach. It was found that the accuracy of the calculations improved as the shapes became larger, since more boundary points were available for calculations, and with a decrease in pixel size. Finally, analytically determined detection capabilities of sensor fields with irregularly shaped sensing coverage regions were compared with Monte Carlo simulations of these regions. In all, three sensing coverage regions were considered, with the analytical and simulated results closely coinciding in all cases.

\section{References}

[1] M. Cardei and J. Wu, "Coverage in wireless sensor networks," in Handbook of Sensor Networks, CRC Press, New York, NY, USA, 2004.

[2] I. F. Akyildiz, D. Pompili, and T. Melodia, "Underwater acoustic sensor networks: research challenges," Ad Hoc Networks, vol. 3, no. 3, pp. 257-279, 2005.

[3] D. Pompili, T. Melodia, and I. F. Akyildiz, "Deployment analysis in underwater acoustic wireless sensor networks," in Proceedings of the 1st ACM international Workshop on Underwater 
Networks (WUWNet '06), pp. 48-55, Los Angeles, Calif, USA, September 2006.

[4] R. J. Urick, Principles of Underwater Sound, Peninsula, Los Altos, Calif, USA, 3rd edition, 1983.

[5] E. F. Golen, N. Shenoy, and B. I. Incze, "Underwater sensor field design using game theory," in Proceedings of the Military Communications Conference (MILCOM '07), October 2007.

[6] B. O. Koopman, Search and Screening: General Principles with Historical Applications, Pergamon, New York, NY, USA, 1980.

[7] J. R. Frost, Principles of Search Theory, Soza, Fairfax, Va, USA, 1999.

[8] B. I. Incze and S. B. Dasinger, "A Bayesian method for managing uncertainties relating to distributed multistatic sensor search," in Proceedings of the 9th International Conference on Information Fusion, July 2006. 

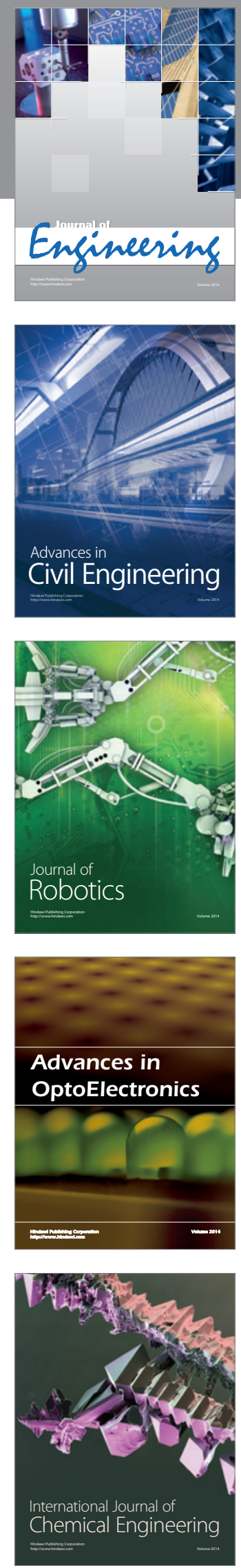

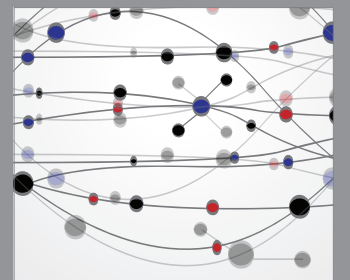

The Scientific World Journal
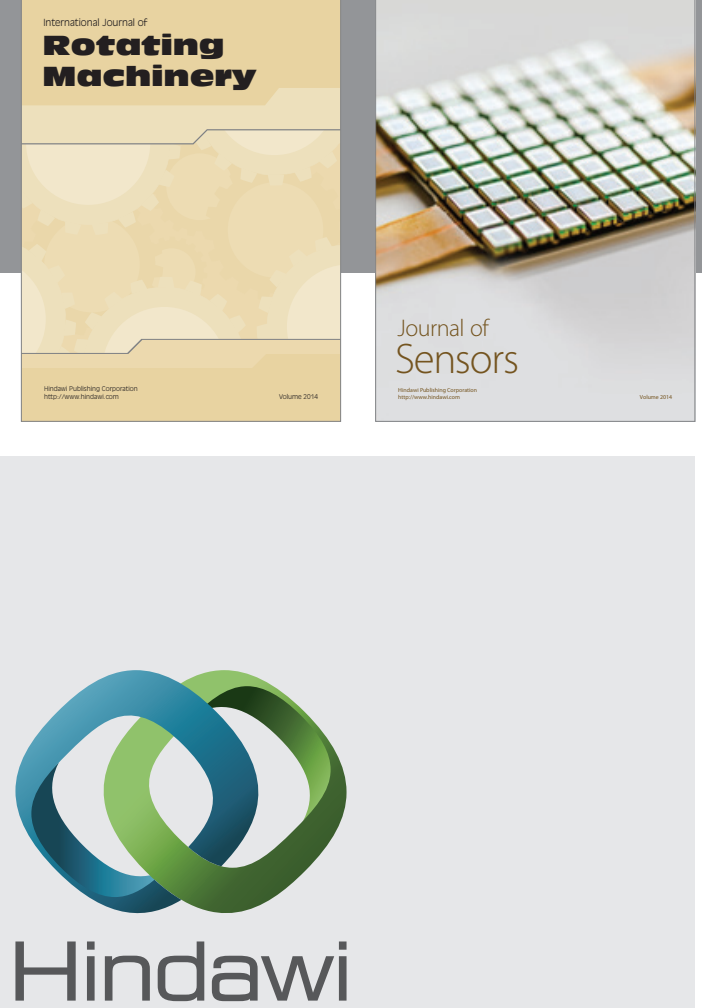

Submit your manuscripts at http://www.hindawi.com
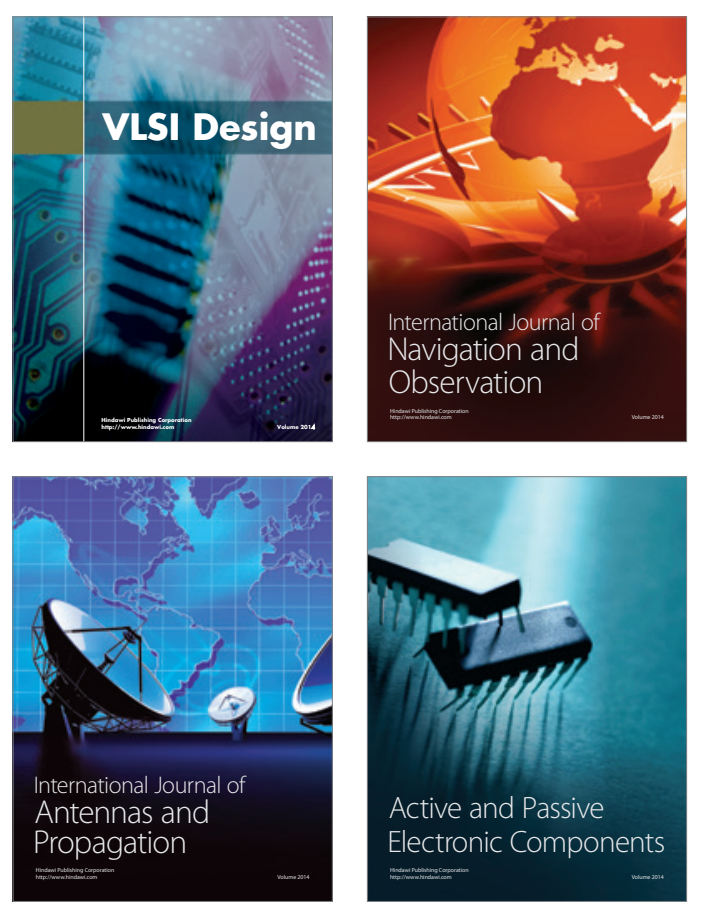
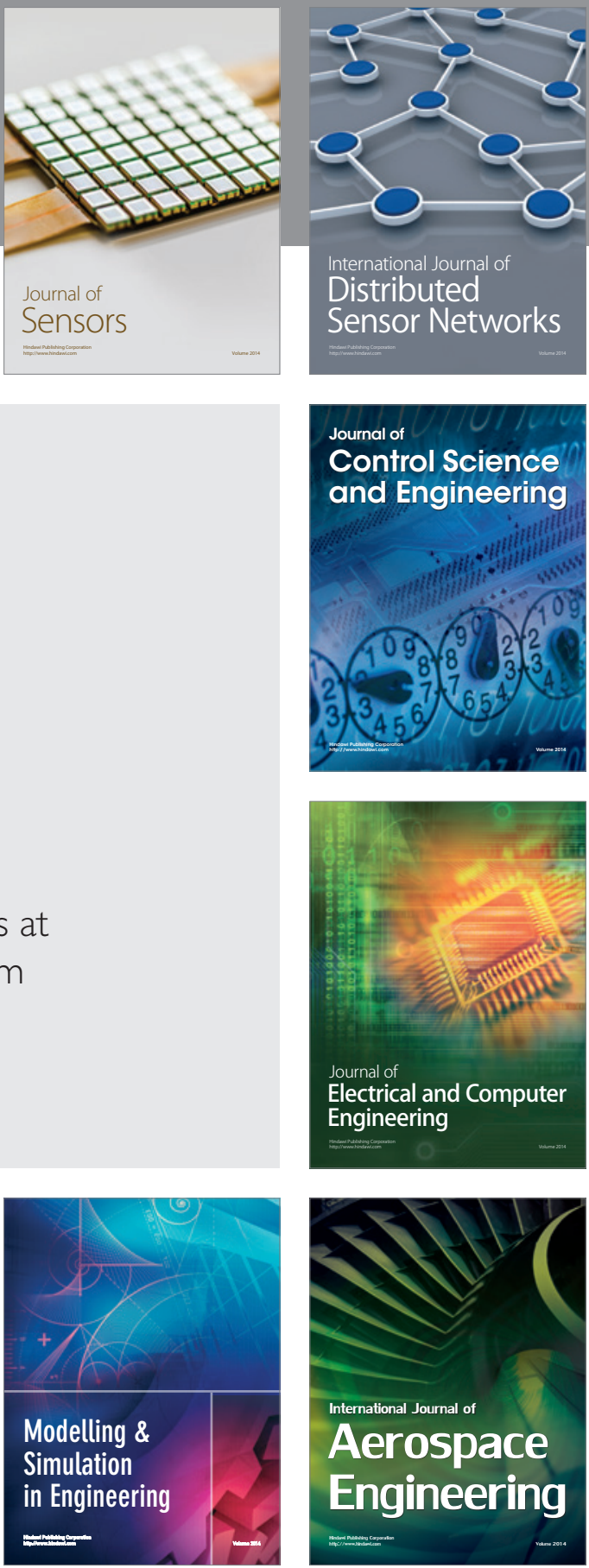

Journal of

Control Science

and Engineering
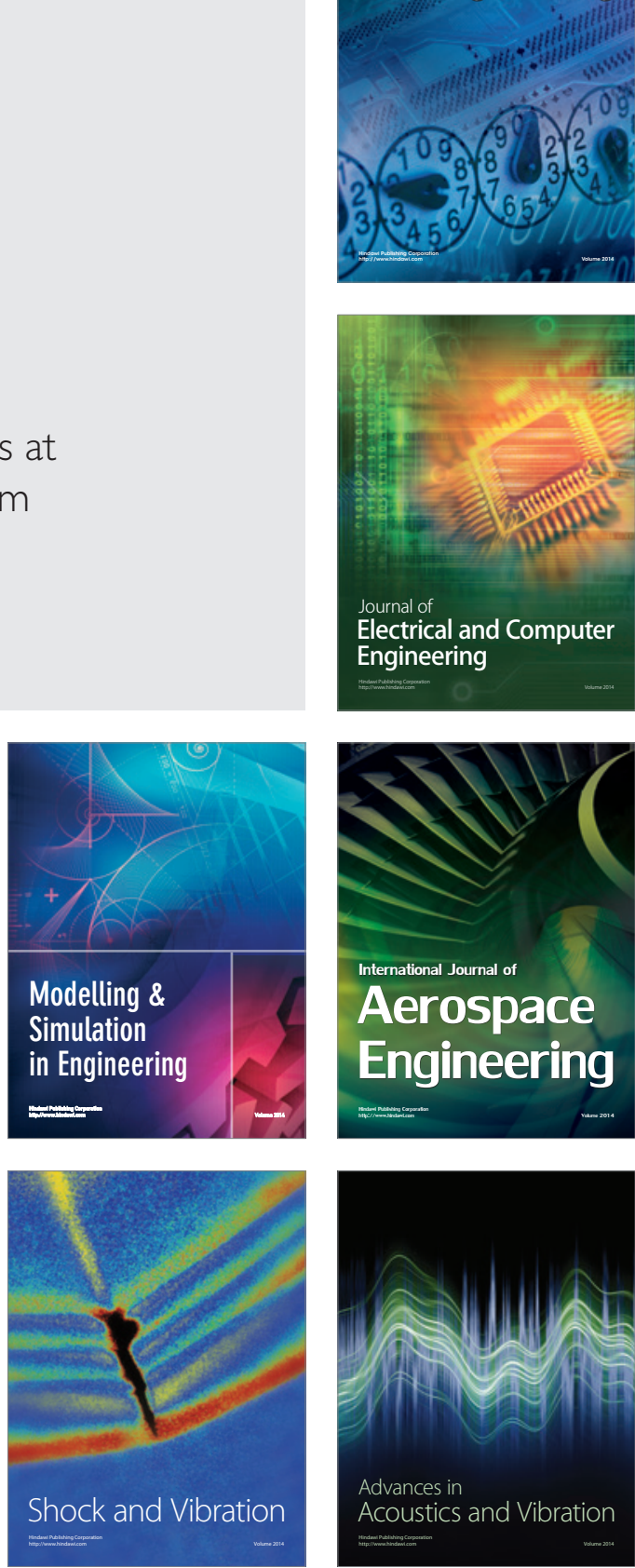